\title{
THE EFFECT OF SMALL VARIATIONS IN PITCH UPON THE INDUCTANCE OF A STANDARD SOLENOID
}

\author{
By Chester Snow
}

\section{ABSTRACT}

A method is described by which the variations in pitch of a single-layer solenoid may be compared with those of the precision lathe screw used to wind it. Assuming that the pitch of the latter is constant, but not known with precision, it is shown how the precision measurements of the length of the windings may be combined with the former measurements to obtain the variation in pitch of the windings relative to an ideal helix whose beginning and end points coincide with the actual one. A mathematical formula for computing the correction $\delta L$ to the inductance due to this variation in pitch is then developed. This formula requires the graphical integration of a certain function times the deformation and practical methods of evaluating it are described. Application is made to observations on two standard solenoids and shows that the correction $\delta L$ to the inductance $L$ may amount to 4 or 5 parts in 100,000 even on the most carefully constructed solenoids. In absolute measurements the correction is, therefore, by no means to be neglected, although it seems hitherto not to have been taken into account in determinations of the ohm.

\section{CONTENTS}

I. Introduction

II. Formula for $\delta L^{2}$

III. Practical method of evaluating the integral for $\delta L$

1. First method.

2. Second method

IV. Numerical examples

1. First example.

2. Second example

V. Summary

VI. Appendix....

\section{INTRODUCTION}

In computing the inductance of a single-layer solenoid, it is at first assumed that the turns of wire are uniformly spaced. Measurements, however, show that this is never the case, no matter how carefully they are constructed. It has been possible by several different methods to measure the very small distance $\delta(y)$, by which a turn at mean distance $y$ from one end is displaced from its ideal position. The ideal position of the nth turn is the position of the nth turn of a uniform solenoid of the same total number of turns, $N$, whose initial and final turns are at the mean position of the initial and final turns of the actual solenoid. The constant pitch of the windings which is used in the formula is defined as the quotient of the measured length of the solenoid by the total number of turns. To the value $L$ of the inductance, computed by any formula which assumes a uniform pitch, we must add a small correction $\delta L$, which represents the increase that would be made in the inductance of the ideal solenoid when its turns 
are displaced by the amounts $\delta(y)$ which deform it into the actual solenoid. These displacements $\delta(y)$ of each circular turn of wire are only in the direction of the axis of the solenoid so far as concerns the present problems. Variations of diameter of the solenoid, as measured over the windings or between turns, which would be due to the elastic deformation of the wires or of the cylindrical form, or of both, have been measured and their influence gives rise to other corrections which do not concern us here. However, for the sake of other possible applications, we formulate the first order correction $\delta L$ caused by any infinitesmal deformation of a circuit.

\section{FORMULA FOR $\delta L$}

Let $L_{0}$ be the inductance of any sort of winding and let $L_{o}+\delta L$ be the inductance when each element of the wire is given a small displacement which is represented by the vector $\delta$, and which varies from point to point on the wire. Let $T_{o}$ and $T_{o}+\delta T$ be the electrokinetic energy in the two cases, the current being one electromagnetic unit in each case. Let $q_{i}$ be the geometric coordinates which determine the configuration of the wire. They are infinite in number and the wire will be treated as a linear circuit. The electromagnetic force (in the generalized Lagrangian sense) tending to increase any such geometric coordinate is $\frac{\partial T}{\partial q_{i}}$ and the work $\delta W$, which is done by all electromagnetic forces during the displacement $\delta$, is therefore $\sum \frac{\partial T}{\partial q_{i}} \delta q_{i}=\delta T$ where $\delta q_{i}$ is the $i^{i h}$ component of the displacement $\delta$ in the Lagrangian sense, and where the current is constant during the displacement. Therefore $\delta T=\delta W$ (with constant current unity). The electrokinetic energy is always given by $T=\frac{1}{2} L I^{2}=\frac{1}{2} L$ (since the current $I=1$ ) so that $\delta T=\frac{1}{2} \delta L=\delta W$, or

$$
\delta L=2 \delta W
$$

That is, the increase, $\delta L$, in the self-inductance of any circuit which is produced by giving it any infinitesimal deformation is equal to twice the work $\delta W$, which is done by its own electromagnetic forces during the deformation with constant current unity. If the vector $H$ is the magnetic field at a point where the element of wire is the vector $d s$ (treating the wire as a linear element), which field is produced by unit current in the wire in its original configuration (omitting the contribution of the element itself), then the electromagnetic force upon $d s$ is the vector product $[d s \times H]$ and the scalar product of this by the vector displacement $([d s \times H] \cdot \delta)=([H \times \delta] \cdot d s)$ is the work done upon the element so that the total work done is

$$
\delta W=\mathcal{S}([d s \times H] \cdot \delta)=\mathcal{S}([H \times \delta] \cdot d s)
$$

integrated with respect to $d s$, along the length of the wire in its initial or unvaried configuration. Combining (1) and (2) gives

$$
\delta L=2 \mathcal{S}([H \times \delta] \cdot d s)=-2 \mathcal{S}([H \times d s] \cdot \delta)
$$


The integral last written represents the total lines of force cut by the displacement. The penalty for idealizing the wire as a linear circuit (with zero cross section) is the same as that incurred in the use of point charges. Since the element's own field does not move it, this singular part of the field must be omitted from $H$. This may be done when the wire is a closely wound coil by taking $H$ as that due to the equivalent uniform distribution of current, such as the equivalent current sheet in the case of a single layer solenoid or helix. In extreme cases, such as that of a very thick wire, the formulation (3) as a line integral would be inadmissible and must be replaced by an analogous volume integral over the volume of the wire.

For the case in hand we replace the single-layer helix by $N$ equal and parallel circular (linear) turns whose centers are on the $y$-axis with the origin $y=0$ at one end. In the initial configuration the circles are equally spaced and the inductance is $L_{0}$. It is then deformed by displacing each turn (center at $y$ ) a distance $\delta(y)$ in the positive $y$-direction where $\delta(y)$ is derived from the observations. It may be positive or negative, and is not only small compared to the distance between two adjacent turns, but also varies slightly from turn to turn. Instead of integrating along the wire, we may then safely take $\frac{N}{l} d y$ (where $l=$ length of windings so that $\frac{N}{l}=$ number of turns per centimeter) as the number of turns in the interval $d y$. Each of these turns is acted upon by a force $-H_{r}(a, y) 2 \pi a$ in the $+y$ direction, where $a$ is the radius of the circular turn and $H_{r}(a, y)$ is the component of magnetic field perpendicular to the axis, which would be produced by the equivalent current sheet around which a current of strength $\frac{N}{l}$ per centimeter along $y$ is circulating right-handedly (each circular turn carries unit current). The equation (3) then gives

$$
\delta L=-\frac{2 N}{l} \int_{0}^{l} H_{r}(a, y) 2 \pi a \delta(y) d y
$$

If $r, \theta$, and $y$ are cylindrical coordinates, the $r$-component of $H$ is a function of $r$ and $y$ only; that is, $H_{r}(r, y)$ and $H_{r}(a, y)$ denotes its value on the sheet where $r=a$. It is continuous at $r=a$. We may compute $H_{\tau}(r, y)$ by recalling that $H$ is the curl of a vector potential $A$, whose cylindrical components $A_{r}$ and $A_{y}$ are zero, while the component $A_{\theta}$ is a function of $r$ and $y$ only. $H_{r}$ is given by

where

$$
H_{r}(r, y)=-\frac{\partial A_{\theta}(r, y)}{\partial y}
$$

$$
A_{\theta}(r, y)=\frac{a N}{l} \int_{0}^{l} d y^{\prime} \int_{0}^{2 \pi} \frac{\cos \theta d \theta}{\sqrt{r^{2}-2 a r \cos \theta+a^{2}+\left(y-y^{\prime}\right)^{2}}}
$$

so that by (5)

$$
\begin{aligned}
H_{r}(r, y) & =-\frac{a N}{l} \int_{0}^{l} d y^{\prime} \frac{\partial}{\partial y} \int_{0}^{2 \pi} \frac{\cos \theta d \theta}{\sqrt{r^{2}-2 a r \cos \theta+a^{2}+\left(y-y^{\prime}\right)^{2}}} \\
& =+\frac{a N}{l} \int_{0}^{l} d y^{\prime} \frac{\partial}{\partial y^{\prime}} \int_{0}^{2 \pi} \frac{\cos \theta d \theta}{\sqrt{r^{2}-2 a r \cos \theta+a^{2}+\left(y-y^{\prime}\right)^{2}}}
\end{aligned}
$$

$49527^{\circ}-31-2$ 
Integrating with respect to $y^{\prime}$ gives

$$
\begin{gathered}
H_{r}(r, y)=\frac{a N}{l} \int_{c}^{2 \pi} \cos \theta d \theta\left\{\frac{1}{\sqrt{r^{2}-2 a r \cos \theta+a^{2}+(l-y)^{2}}}\right. \\
\left.-\frac{1}{\sqrt{r^{2}-2 a r \cos \theta+a^{2}+y^{2}}}\right\}
\end{gathered}
$$

Placing $r=a$ gives

$$
-H_{r}(a, y)=\frac{2 \pi N}{l}\left[\varphi_{1}(\mu)-\varphi_{1}\left(\mu^{\prime}\right)\right]
$$

where

$$
\mu^{2}=\frac{1}{1+\left(\frac{y}{2 a}\right)^{2}} \text { and } \mu^{\prime 2}=\frac{1}{1+\left(\frac{l-y}{2 a}\right)^{2}}
$$

and

$$
\pi \varphi_{1}(\mu) \equiv-\mu \int^{\pi / 2} \frac{\cos 2 \theta d \theta}{\sqrt{1-\mu^{2} \sin ^{2} \theta}}=\frac{2}{\mu}[K(\mu)-E(\mu)]-\mu K(\mu)
$$

where $K$ and $E$ are the complete elliptic integrals of the first and second kind of modulus $\mu$. The formula (4), therefore, becomes by the use of (8)

$$
\delta L=\frac{8 \pi^{2} a N^{2}}{l^{2}} \int_{0}^{l} \delta(y)\left[\varphi_{1}(\mu)-\varphi_{1}\left(\mu^{\prime}\right)\right] d y
$$

where $\mu$ and $\mu^{\prime}$ are functions of $y$ given by (9), and of course $\delta(y)$ is the observed function of $y$. We may replace the latter by a function $f_{1}(y)$ defined by

$$
f_{1}(y)=\delta(y)-\delta(l-y)
$$

The equation (11) then transforms into

$$
\delta L=\frac{8 \pi^{2} a N^{2}}{l^{2}} \int_{0}^{l} f_{1}(y) \varphi_{1}(\mu) d y
$$

It is next convenient to introduce a new variable of integration by the transformation

$$
\lambda \equiv \frac{y}{2 a}
$$

If we define $f(\lambda)$ as $f_{1}(2 a \lambda)$ and $\psi(\lambda)$ by

$$
\psi(\lambda) \equiv \pi \phi_{1}(\mu) \text { where } \mu^{2}=\frac{1}{1+\lambda^{2}}
$$

then (13) finally becomes

$$
\delta L=4 \pi\left(\frac{N}{\lambda_{m}}\right)^{2} \int_{0}^{\lambda_{m}} f(\lambda) \psi(\lambda) d \lambda
$$

where

$$
\lambda_{m} \equiv \frac{l}{2 a}=\text { the maximum value of } \lambda
$$


The deformation of the helix is represented in (16) by the small observed function $f(\lambda)$ which may be plotted as a function of $\lambda$ from $\lambda=0$ to $\lambda=\lambda_{m}$. The function $\psi(\lambda)$ defined by (10) and (15) has been computed by the use of tables of the elliptic integrals and plotted in Figure 1 from $\lambda=0.07$ to $\lambda=5.0$. Since it becomes logarithmically infinite at $\lambda=0$, the equation (16) needs some reshaping in order to become practicable for the evaluation of $\delta L$ by graphical integration.

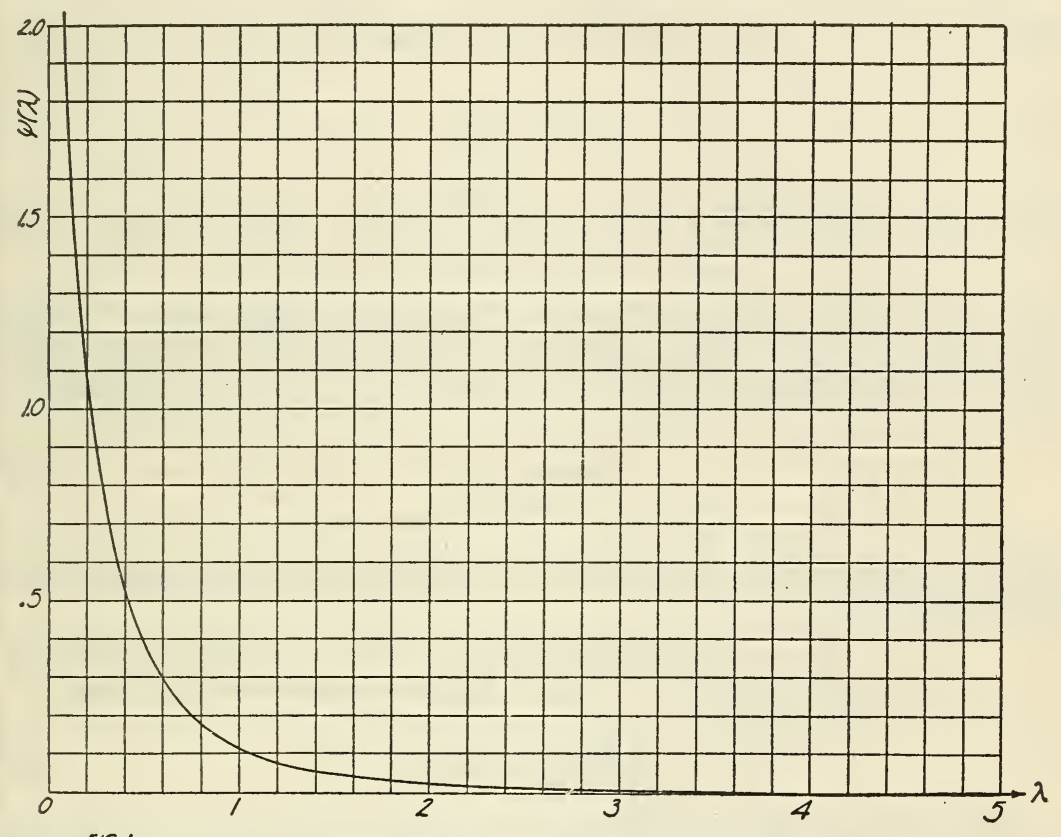

176\%.

FIGURE 1.-The function $\psi(\lambda)$

\section{PRACTICAL METHOD OF EVALUATING THE INTEGRAL FOR $\delta L$}

\section{FIRST METHOD}

Evidently a positive value $\epsilon$ may always be so chosen that the curve of observation $f(\lambda)$ may be represented by

$$
f(\lambda)=f(o)+f^{\prime}(o) . \quad \lambda \text { in the range } o \bar{\Sigma} \lambda \bar{\equiv} \epsilon
$$

so that (16) may be written

$$
\left.\delta L=4 \pi\left(\frac{N}{\lambda_{m}}\right)^{2}\left\{f(0) \int_{0}^{\epsilon} \psi(\lambda) d \lambda+f^{\prime}(0) \int_{0}^{\epsilon} \lambda \psi(\lambda) d \lambda\right)+\int_{\epsilon}^{\lambda_{m}} f(\lambda) \psi(\lambda) d \lambda\right\}
$$

For the last integral the integrand is easily plotted and is finite, and may be evaluated by graphical integration. The first two integrals are finite although the integrand becomes infinite in the first, but both may be evaluated analytically by means of two integral formulas 
which are given in Bureau of Standards Scientific Paper No. 537 on page 449 , which are

$$
\begin{aligned}
-\pi \int \frac{\phi_{1}(\mu) d \mu}{\mu^{2} \sqrt{1-\mu^{2}}}=\frac{\sqrt{1-\mu^{2}}}{\mu^{2}}\{K(\mu)-E(\mu)\} \\
-\pi \int \frac{\phi_{1}(\mu) d \mu}{\mu^{3}}=\frac{1}{3 \mu}\left\{\frac{2\left(1-\mu^{2}\right)}{\mu^{2}}[K(\mu)-E(\mu)]-E(\mu)\right\}
\end{aligned}
$$

Since $\pi \phi_{1}(\mu)=\psi(\lambda)$, and since the dependence of $\mu$ upon $\lambda$ becomes

$$
\mu^{2}=\frac{1}{1+\lambda^{2}}
$$

it is evident that $-\frac{d \mu}{\mu^{3}}=\lambda d \lambda$ and $\frac{-d \mu}{\mu^{2} \sqrt{1-\mu^{2}}}=d \lambda$. The left sides of (20)

and (21) become $\int \psi(\lambda) d \lambda$ and $\int \lambda \psi(\lambda) d \lambda$, respectively. Taking the definite integrals from $\lambda=0$ to $\lambda=\epsilon$ (noting that for the particular function considered $f(o)=0$ ), we find from (19) after placing

$$
7 \equiv \frac{1}{\sqrt{1+\epsilon^{2}}}
$$

the final formula for computing the increase $\delta L$ in inductance due to the deformation.

$$
\begin{gathered}
\delta L=4 \pi\left(\frac{N}{\lambda_{m}}\right)^{2}\left\{\frac{1}{3}\left[1+\frac{2\left(1-k^{2}\right)}{k^{3}}(K(k)-E(k))-\frac{E(k)}{k}\right] f^{\prime}(0)\right. \\
\left.+\int_{\epsilon}^{\lambda_{m}} \psi(\lambda) f(\lambda) d(\lambda)\right\}
\end{gathered}
$$

Assuming that the straight line representation (18) of $f(\lambda)$ will generally be possible for the range $0<\lambda<\epsilon=0.1$, this becomes

$$
\delta L=4 \pi\left(\frac{N}{\lambda_{m}}\right)^{2}\left\{0.011 f^{\prime}(0)+\int_{\epsilon}^{\lambda_{m}} \psi(\lambda) f(\lambda) d \lambda\right\}
$$

To compute $\delta L$ by this formula, it will be recalled that $N$ is the total number of turns each of radius $a$ and that $\lambda$ is $\frac{y}{2 a}$ and $\lambda_{m}$ is $\frac{l}{2 a}$ where the length of the solenoid is $l \mathrm{~cm}$ and $y$ is the distance in $\mathrm{cm}$ from one end to a variable point and the method of constructing and plotting the small function $f(\lambda)$ is as follows: The observed displacement in $\mathrm{cm}$ relative to the ideal helix of a turn at distance $y$ from one end being the small function of $y, \delta(y)$, (which is reckoned as positive when the displacement is in the direction of increasing $y$ ), we define $f_{1}(y)$ by $f_{1}(y)=\delta(y)-\delta(l-y)$ and then make the transformation $y=2 a \lambda$ representing the function $f_{1}(y)=f_{1}(2 a \lambda)$ by the new symbol $f(\lambda)$. This may be plotted as a function of $\lambda$ and the ordinates when multiplied by the ordinates of the curve Figure 1 for $\psi(\lambda)$ give the integrand of the integral in (24) or (24a). If this integrand, $\psi(\lambda) f(\lambda)$, be plotted as a function of $\lambda$, the area under the curve can be found by 
graphical integration and represents the integral desired in (24). Since $\lambda$ is dimensionless and $\delta(y)$ and $f(\lambda)$ and $f^{\prime}(\lambda)$ are given in centimeters, the change of inductance $\delta L$ given by formulas (16), (24) or (24a) will be measured in absolute c. g. s. electromagnetic units or centimeters. $\quad\left(1 \mathrm{~cm}=10^{-9}\right.$ henries.)

\section{SECOND METHOD}

The procedure outlined above is applicable when $f(\lambda)$ is any function which is finite and has a finite slope at $\lambda=0$. If $f(o)$ is not zero, an improper integral is involved and this method of evaluating the integral analytically over a small initial range must be used as graphical integration could give no certainty in the case of a curve going to infinity at $\lambda=0$. Since, however, the function $f(\lambda)$ always vanishes at $\lambda=0$ and has a finite initial slope, the integrand $\psi(\lambda) f(\lambda)$ is always finite (in fact zero) at $\lambda=0$, and there is no practical difficulty in evaluating it graphically over the entire range. The practical difficulty is in plotting it right down to the point $\lambda=0$. This may be overcome by obtaining an asymptotic expansion for $\psi(\lambda)$ in the neighborhood of $\lambda=0$. Such an expansion is given in Bureau of Standards Scientific Paper No. 537 on page 449, formula (56) by which

$$
\pi \Phi_{1}(\mu)=\left[1+\frac{3}{4}\left(1-\mu^{2}\right)\right] \ln \frac{4}{\sqrt{1-\mu^{2}}}-2-\frac{3}{4}\left(1-\mu^{2}\right)
$$

in which additive terms of the order of $\left(1-\mu^{2}\right) \log \frac{1}{1-\mu^{2}}$ have been neglected. Since $1-\mu^{2}=\frac{\lambda^{2}}{1+\lambda^{2}}$ this becomes

$$
\psi(\lambda)=\left(1+\frac{3}{4} \lambda^{2}\right) \ln \frac{4}{\lambda}-2-\frac{1}{4} \lambda^{2}
$$

which may be used for values of $\lambda$ less than 0.1 with an absolute error which is then always less than $0.1^{4} \ln 10=0.00023$.

If we use (25) for computing the integrand $\psi(\lambda) f(\lambda)$ of equation (16) in the small range, say, $0<\lambda<0.1$ then formula (16) may be readily evaluated graphically.

In the first example below, the curve for $f(\lambda)$ is practically a straight line through the origin for $0<\lambda<0.1$ and the formula (24a) has been found most convenient. In the second example, the second method is used.

Before taking up these numerical examples, the method should be described by which the value of $\delta(y)$ was inferred from the observations. As this method was somewhat indirect, new measurements are being considered by which $\delta(y)$ may be measured directly with reference to a standard of length by means of a cathetometer.

We define the ideal solenoid of constant pitch as one of the same number $N$ of turns whose beginning and end coincide with the actual one. Taking the $y$-axis as the axis of the cylinder with the origin at one end, let $\delta(y)$ be the small displacement in the $y$-direction which deforms the element of the ideal winding, which is distant $y$ from the end of the coil into its actual position. Evidently $\delta(y)$ is zero at both ends; that is

$$
\delta(0)=\delta(l)=0
$$


Since the cylindrical form is of quartz, it may be assumed to have the same length $l$ when mounted on the lathe for winding and comparison with the screw as it was when finally set up for measurement of its length and diameter.

The nearest substitute for the ideal coil in terms of which the actual one may be calibrated is the screw. The displacement $\Delta(y)$ which would deform an element of the screw thread into the position of the corresponding element of the actual winding was determined as a function of $y$ by means of an optical lever on the carriage which made contact with the side of the wire. As the coil was slowly rotated and the lever carried forward on the screw, four observations were taken for each turn $-4,000$ readings in the case of the quartz cylinder with 1,000 turns. The scale deflections, multiplied by the calibration constant of the lever, gave $\Delta(y)$. These measurements were taken immediately after winding, with the cylinder geared to the screw exactly as in the windings, so that the element of winding was compared with practically the same part of the screw that was used in winding it. Hence, if the wire had not rolled in going on to the cylinder its pitch should have possessed practically the same variations as the screw and no deflection of the optical lever should have been observed in moving from one end to the other, even though the screw were variable in pitch. Deflections $\Delta(y)$ were observed, however, and these were reasonably concordant with a similar set of observations (which were taken on one coil) in which the optical lever was replaced by a micrometer microscope The value of $\Delta(y)$ was sometimes positive and sometimes negative, but never more than 2 per cent of the pitch or distance between two turns, which was $0.1 \mathrm{~cm}$ in the case of the quartz cylinder of 1,000 turns, whose length was very approximately $100 \mathrm{~cm}$. The displacements $\delta(y)$ and $\Delta(y)$ are reckoned as positive when they are in the direction of increasing $y$.

If we let $\Delta_{\mathrm{s}} y$ denote the displacement (in the $+y$ direction) which would deform the ideal helix into the screw, then

$$
\delta(y)=\Delta(y)+\Delta_{s}(y)
$$

Now if the screw is assumed to have a constant but unknown pitch at the time of comparison, then

$$
\Delta_{s}(y)=A+B y \text { where } A \text { and } B \text { are constants }
$$

the latter depending upon the temperature. A change in the value of $A$ merely shifts the screw as a rigid body and is irrelevant. Combining (27) and (28) gives

$$
\delta(y)=\Delta(y)+A+B y
$$

The constants $A$ and $B$ are determined by the two conditions (26) that $\delta(0)=\delta(l)=0$. They are

$$
\begin{aligned}
& A=-\Delta(o) \\
& B=\frac{1}{l}[\Delta(o)-\Delta(l)]
\end{aligned}
$$

so that (29) becomes

$$
\delta(y)=\Delta(y)-\left[\frac{\Delta(l)-\Delta(o)}{l} y+\Delta(o)\right]
$$


The observations are given in the form of a curve in which is plotted as a function of $y$ from $y=0$ to $y=l$, the small observed function $\Delta(y)$, which is the displacement of the winding relative to the screw. The equation (30) then gives the desired function $\delta(y)$ representing the displacement which deforms the ideal solenoid into the actual one. The curve for $\delta(y)$ may be plotted without computation by drawing a straight line through the end points of the curve for $\delta(y)$. This is the straight line whose ordinates are represented by the bracket which is subtracted from $\Delta(y)$ in equation (30). Hence, the vertical distance of the curve for $\Delta(y)$ above this straight line represents $\delta(y)$. (See fig. 2.)

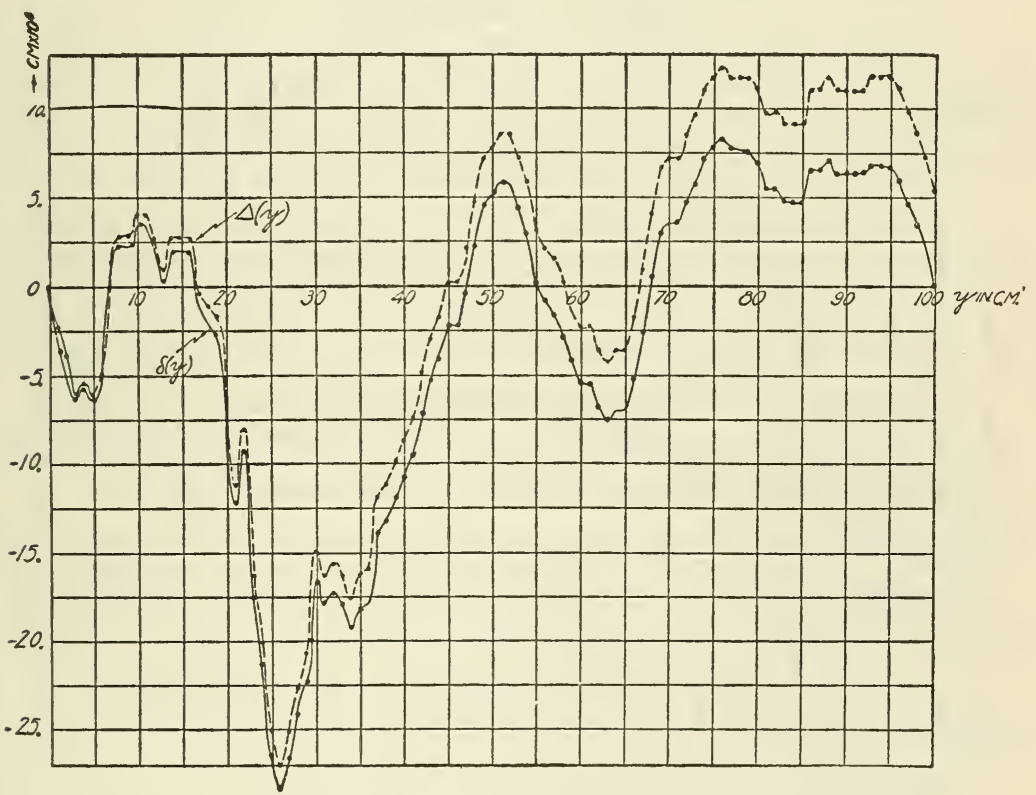

FIgURE 2.-The observed variations in pitch of coil No. 1

\section{NUMERICAL EXAMPLES}

\section{FIRST EXAMPLE}

This is the case of a single layer helix of 1,000 turns of length $l=100 \mathrm{~cm}$ wound upon a quartz cylinder whose radius $a$ is $14 \mathrm{~cm}$, so that $N=1,000$ and $\lambda_{\mathrm{m}}=\frac{100}{28}=3.57$ and formula (24a) becomes

$$
\delta L=\frac{4 \pi(10)^{6}}{(3.57)^{2}}\left\{0.011 f^{\prime}(o)+\int_{.1}^{3.57} 4(\lambda) f(\lambda) d \lambda\right\}
$$

The observed variation in pitch is shown in Figure 2 by the curve which gives $\Delta(y)$ the displacement which transforms the screw thread into the actual winding. The curve in this figure for $\delta(y)$ was obtained graphically as explained since $\delta(y)$ is the height of the $\Delta(y)$ curve above the straight line connecting the ends of the latter. The $\delta(y)$ curve represents the displacement which would deform the ideal helix into the actual windings. From $\delta(y)$ the function $f_{1}(y)=$ 
$\delta(y)-\delta(l-y)$ was constructed and plotted in Figure 3 as $f(\lambda)$ where $\lambda=\frac{y}{28}$. In Figure 3 is also shown the integrand $\psi(\lambda) f(\lambda)$, the factor $\psi$ being taken from the curve of Figure 1. The area under the latter curve of Figure 3 was found by use of the integraph to be

$$
\int_{0.1}^{3.57} \psi(\lambda) f(\lambda) d \lambda=-4.7(10)^{-4} \text { with error }<1 \text { per cent of itself. }
$$

The unit is here the centimeter of inductance $\left(10^{-9}\right)$, not area on the paper. The initial slope of the curve $f(\lambda)$ in Figure 3 is

$$
f^{\prime}(0)=-105\left(10^{-4}\right) \mathrm{cm}
$$

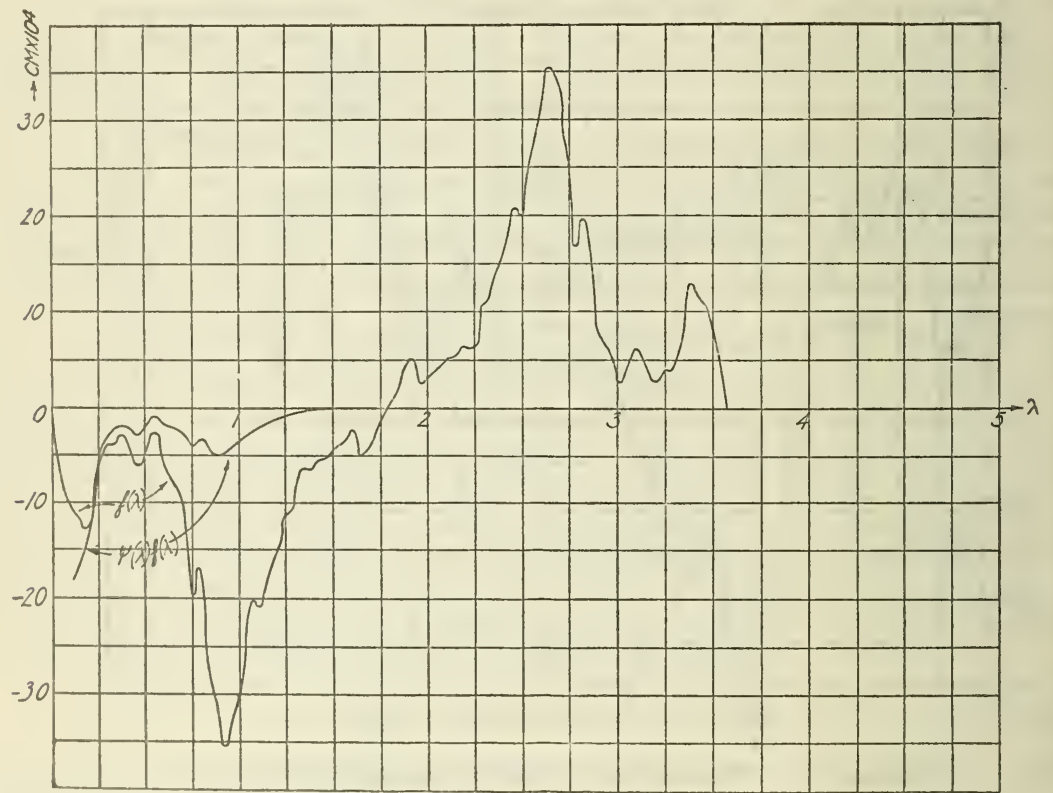

FIgURE 3.-The functions $f(\lambda)$ and $\psi(\lambda) f(\lambda)$ for coil No. 1

Hence formula (24) becomes

$$
\delta L=-\frac{400 \pi}{(3.57)^{2}}\{1.15+4.7\}=-580 \mathrm{~cm}
$$

which must be subtracted from the value $L_{o}$ which is computed on the assumption of constant pitch. Since $L_{0}$ is approximately $68 \times 10^{6} \mathrm{~cm}$, this correction is about 8 parts in $1,000,000$.

\section{SECOND EXAMPLE}

This is the case of a single-layer helix of 270 turns with the same nominal pitch $(0.1 \mathrm{~cm})$ as in the preceding case, but wound upon a cylinder of pyrex glass, the mean diameter of the coil being $2 a=44.56$ $\mathrm{cm}$, so that since $l=27 \mathrm{~cm}, \lambda_{m}=\frac{27}{44.56}=0.605$. In this case there were two types of observations whose discrepancy served to indicate the 
Snow]

Effect of Variations in Pitch on Inductance

787

probable total error of the method. The first was by means of the optical lever; the second was by means of readings taken with a micrometer microscope. The two sets of data are shown in Figure 4,

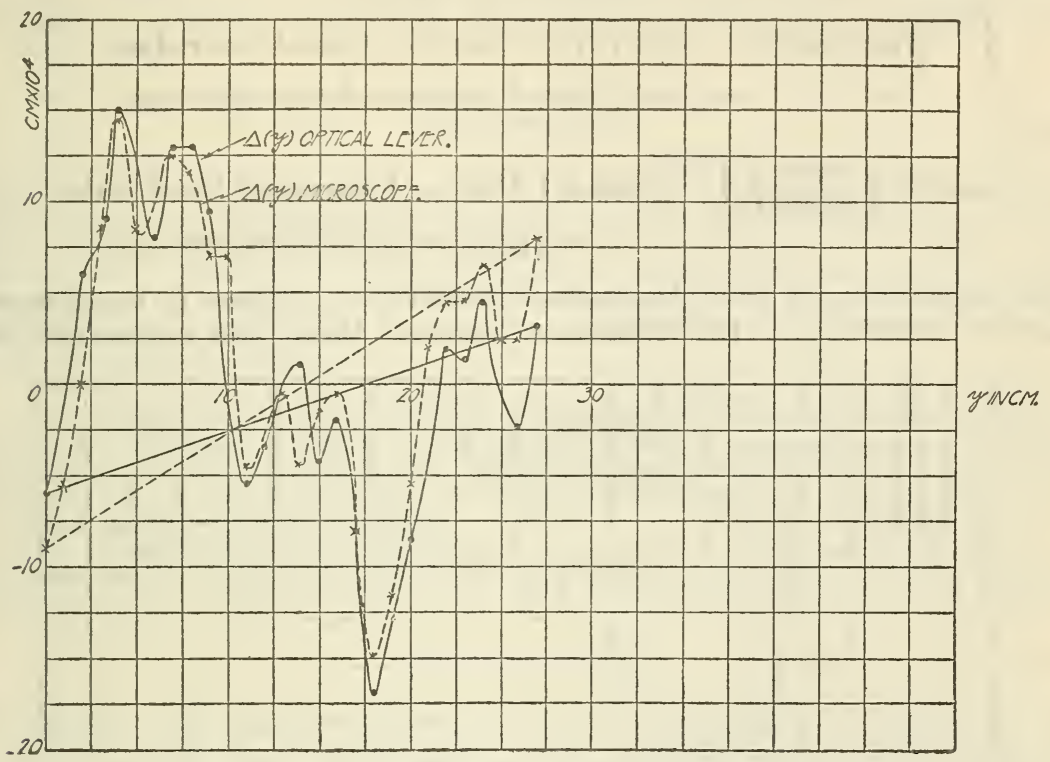

FIGURE 4. -Observed variations in pitch of coil No. 2

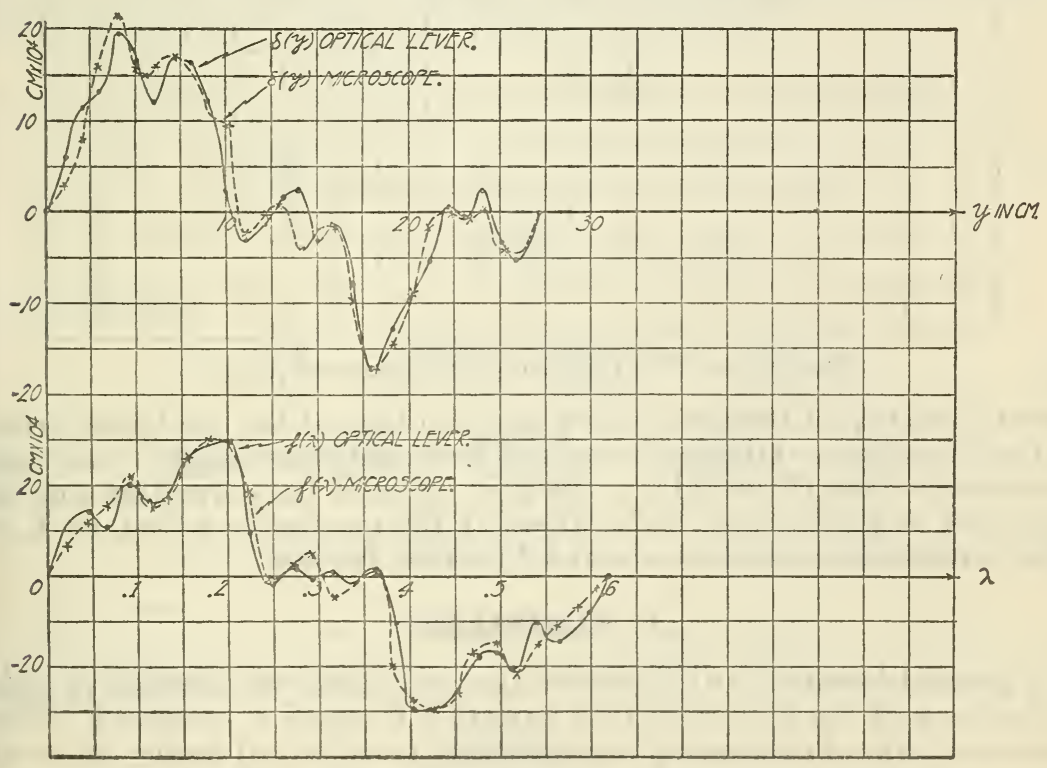

Figure 5. -The functions $\delta(\lambda)$ and $f(\lambda)$ for coil No. 2

which gives $\Delta y$ in the two cases. The corresponding $\delta(y)$ 's were constructed graphically and shown in the upper curves of Figure 5 and the $f(\lambda)$ 's in the lower curves. In Figure 6 are shown the two 
curves for $\psi(\lambda) f(\lambda)$, these being constructed by the use of formula (25) for values of $\lambda$ less than 0.1 . The area under these two curves was found by the use of an integraph and gave

$$
\begin{aligned}
\int_{0}^{0.605} \psi(\lambda) f(\lambda) d \lambda & =+4.65(10)^{-4} \mathrm{~cm} \text { for optical lever data } \\
& =+4.47(10)^{-4} \mathrm{~cm} \text { for microscope data }
\end{aligned}
$$

Hence

$$
\begin{aligned}
\delta L=4 \pi\left(\frac{270}{0.605}\right)^{2} \int_{0}^{0.605} \psi f d \lambda & =1,160 \mathrm{~cm} \text { for optical lever data } \\
& =1,120 \mathrm{~cm} \text { for microscope data }
\end{aligned}
$$

(The units here are in centimeters of inductance.) Since $L_{o}$ is approximately $26(10)^{6} \mathrm{~cm}$, the difference between these two corrections is

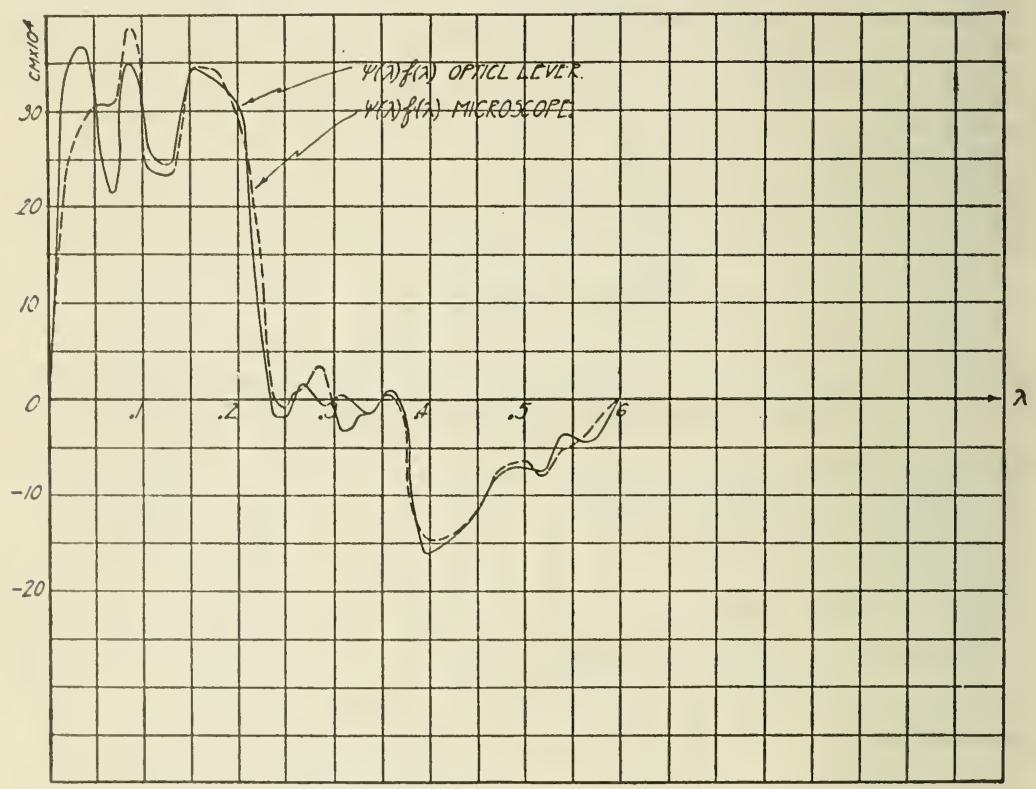

Figure 6.-The function $\psi(\lambda) f(\lambda)$ for coil No. 2

about 2 parts in $1,000,000$, which gives an idea of the probable error in the correction. Giving the optical lever data the weight 2 and the microscope data the weight 1 , the best value of the correction due to variation in pitch seems to be $\delta L=+1,150 \mathrm{~cm}$ (to be added to $L_{o}$ ). This correction amounts to about 4.4 parts in 100,000.

\section{SUMMARY}

$\Lambda$ general formula (16) is derived for computing the increase in selfinductance of the helix when its variation in pitch is observed. The practical form of evaluating it graphically is explained under (24) and (24a), and also in connection with equation (25). The directions for constructing the observational function are summarized after formula (24a). The application shows that this correction may amount to 4 or 5 parts in 100,000 , which shows that it must be taken account of in absolute measurements which use the coils as standards whose inductance may be computed from their geometric dimensions, 


\section{APPENDIX}

\section{AN INDEPENDENT METHOD OF DERIVING THE EFFECT OF SMALL VARIATIONS IN PITCH ON THE INDUCTANCE OF A STANDARD SOLENOID}

(The following method was derived by F. W. Grover during the editorial reading of the preceding paper)

The inductance of a coil may be obtained by summing the self-inductances of the turns and the mutual inductances of all the pairs of turns of which the coil is composed. We will assume, as has been done in the preceding paper, that the coil is made up of parallel, coaxial, circular turns of wire; that is, the helicity will be neglected. Thus the effect of irregularities in the winding pitch will be taken into account by supposing each turn to be displaced in the axial direction by an amount equal to the average of the displacements of all the elements of the turn from their ideal positions.

It is evident that the self-inductance of an individual turn will not be altered by such a displacement. The mutual inductances of the various pairs of turns are, on the contrary, changed by the displacements of the wires from their ideal positions, and the algebraic sum of the changes of mutual inductance of all the pairs of turns of the coil will give the change of inductance of the coil, due to the assumed irregularities of winding.

If two turns, whose axial coordinates are $y$ and $(y+\Delta y)$ in the ideal coil are given displacements $\delta_{1}$ and $\delta_{2}$, respectively, their mutual inductance suffers a change equal to

$$
\delta M=-\left(\delta_{1}-\delta_{2}\right) \frac{d M}{d y}
$$

Furthermore, $\frac{d M}{d y}$ will be the same for all pairs of turns which are equally spaced in their ideal positions. Applying these considerations to the observed displacements $\delta_{1}, \delta_{2}, \delta_{3},-\ldots \delta_{n}$ of all the turns of a coil of $N$ turns, wound with an average pitch $g$, the inductance of the coil is changed by an amount

$$
\begin{gathered}
\delta L=\Sigma \delta M=-2\left[\left(\frac{d M}{d y}\right)_{1}\left\{\left(\delta_{1}-\delta_{2}\right)+\delta_{2}-\delta_{3}\right)+\cdots+\left(\delta_{n-1}-\delta_{n}\right)\right\} \\
+\left(\frac{d M}{d y}\right)_{2}\left\{\left(\delta_{1}-\delta_{3}\right)+\left(\delta_{2}-\delta_{4}\right)+\cdots+\left(\delta_{n-2}-\delta_{n}\right)\right\} \\
\left.+\cdots+\left(\frac{d M}{d y}\right)_{n-1}\left(\delta_{1}-\delta_{n}\right)\right]
\end{gathered}
$$

or, rearranging

$$
\begin{aligned}
& \delta L=-2\left[\left(\delta_{1}-\delta_{n}\right)\left\{\left(\frac{d M}{d y}\right)_{1}+\left(\frac{d M}{d y}\right)_{2}+\cdots+\left(\frac{d M}{d y}\right)_{n-1}\right\}\right. \\
& +\left(\delta_{2}-\delta_{n-1}\right)\left\{\left(\frac{d M}{d y}\right)_{2}+\left(\frac{d M}{d y}\right)_{3}+\cdots+\left(\frac{d M}{d y}\right)_{n-2}\right\}+\cdots \\
& \left.+\left(\delta_{r}-\delta_{n-r+1}\right)\left\{\left(\frac{d M}{d y}\right)_{r}+\left(\frac{d M}{d y}\right)_{r+1}+\cdots+\left(\frac{d M}{d y}\right)_{n-r}\right\}\right]
\end{aligned}
$$

in which $\left(\frac{d M}{d y}\right)_{p}$ is the rate of change of the mutual inductance with respect to their axial distance, apart, of two turns separated by a distance $p g$. The final term in (3) is

$$
\left(\delta_{\frac{n}{2}}-\delta_{\frac{n}{2}+1}\right)\left(\frac{d M}{d y}\right)_{\frac{n}{2}}, \text { if } N \text { is even }
$$

and

$$
\left(\delta_{\frac{n-1}{2}}-\frac{\delta_{n+3}}{2},\left(\frac{d M}{d y}\right)_{\frac{n-1}{2}} \text {, if } N\right. \text { is odd. }
$$


The calculation of $\delta L$ is thus seen to depend upon the calculation of $\left(\frac{d M}{d y}\right)$ for pairs of coaxial circular filaments, with axial separations $g, 2 g$, $3 g$, etc. Since the force between two filaments carrying unit current is $-\left(\frac{d M}{d y}\right)$, the terms of cquations (2) and (3) are seen to represent work done by the electromagnetic forces during the deformation from the ideal winding, a result agreeing with equation (1) of the text.

Except for turns very close together, the values of the $\left(\frac{d M}{d y}\right)$ can not be expressed by a single series expression. However, the well-known elliptic integral formula of Maxwell, Elect. and Mag. volume 2, page 339, is universally applicable, or more conveniently, numerical values may be interpolated from the tables of Nagaoka and Sakurai, Institute of Physical and Chemical Research, Table 2, Tokyo, 1927.

The calculation of $\delta L$ for short coils, or coils of oniy a few turns may readily be carried out by the use of formula (3) and such tables. For a coil of many turns, however, this process would become very laborious. In such cases the winding pitch is small compared with the dimensions of the coil, and $\left(\frac{d M}{d y}\right)$ changes slowly with the spacing of the turns. Thus it is possible to transform the summation of equation (3) into an integration over the length of the coil, and it will be shown that this leads to Doctor Snow's equation (13).

To prove this, we note that the displacements of the turns enter in (3) in the form $\delta(y)-\delta(l-y)=f_{1}(y)$, so that on summing along the coil, under the assumption that $\Delta y=g$ is very small, each term of (3) takes the form $\frac{1}{g}[\delta(y)-\delta(l-y)]\left(M_{y}-\right.$ $\left.M l-{ }_{y}\right)$, where $M_{y}$ and $M_{l}-{ }_{y}$ are the mutual inductances of equal coaxial circles, - separated by axial distances $y$ and $(l-y)$, respectively.

Thus, if we remeinber that the winding density is $\frac{N}{l}$ turns per $\mathrm{cm}$. of axial length, equation (3) becomes

$$
\delta L=\frac{2}{g} \int_{0}^{y_{2}} \frac{N}{l} f_{1}(y)\left(M_{y}-M_{\tau_{-}}\right) d y
$$

It is, however, to be noted that the function $\pi \varphi_{1}(\mu)$, of equation (15), is a factor in Maxwell's elliptic integral formula for the mutual inductance of equal coaxial circles (loc. cit.), so that we may write

and, therefore,

$$
\frac{M_{y}}{4 \pi a}=\psi(\lambda)
$$

$$
\delta L=\frac{8 \pi N a}{g l} \int_{0}^{l / 2} f_{1}(y)\left[\psi(\lambda)-\psi\left(\lambda_{m}-\lambda\right)\right] d y
$$

The second integral in this equation may be transformed by changing the variable to $y^{\prime}=l-y$, so that it takes the form $\int_{\frac{l}{2}}^{l} f_{1}(y) \psi(\lambda) d y$. Thus $(7)$ becomes finally

$$
\delta L=\frac{8 \pi a N^{2}}{l^{2}} \int_{0}^{l} f_{1}(y) \psi(\lambda) d y
$$

which is exactly the equation (13) of the paper, since $\pi \varphi_{1}(\mu)=\psi(\lambda)$.

The alternative method is thus seen to lead to exactly the same correction formula for the inductance change due to variations in winding pitch as was found by snow's more general solution of the problem in the preceding paper.

Washington, September 15, 1930. 